\title{
Gamificação aplicada ao ensino e aprendizagem de Engenharia de Software: Um mapeamento sistemático
}

\author{
Matheus B. Altomar ${ }^{1}$, Filipe B. Furtado ${ }^{1}$, Felipe T. Cazetta ${ }^{1}$, \\ Leonardo H. Silva ${ }^{1}$, Alessandreia M. de O. Julio ${ }^{1}$ \\ ${ }^{1}$ Departamento de Ciência da Computação - UFJF) \\ \{matheus.altomar, brinati, fterrana
leonardoholanda, alessandreia.oliveira\}@ice.ufje.br
}

\begin{abstract}
A gamificação é considerada um recurso útil em diversas áreas. Particularmente, entre seus objetivos na educação estão a redução da evasão e o aumento da motivação nas disciplinas e no curso. Por outro lado, muitas das tarefas da disciplina de Engenharia de Software são demoradas e podem, ser consideradas repetitivas pelos alunos. Diante disso, este artigo descreve um mapeamento sistemático sobre a aplicação da gamificação no ensino e aprendizagem de Engenharia de Software. As 17 publicações resultantes foram usadas para responder às questões de pesquisa propostas e mostraram que a gamificação tem resultados positivos quando aplicada à Engenharia de Software.
\end{abstract}

\begin{abstract}
Gamification is considered a useful resource in several areas. Particularly, among its goals in education are reducing dropout and increasing motivation in subjects and in the course. On the other hand, many of the tasks in the discipline of Software Engineering are time consuming and can sometimes be considered repetitive by students. Therefore, this paper describes a systematic mapping on the application of gamification in the teaching and learning of Software Engineering. The 17 resulting publications were used to answer the proposed research questions. Such publications show that gamification has positive results when applied to Software Engineering.
\end{abstract}

\section{Introdução}

A forma de ensinar Engenharia de Software (ES) nos cursos de graduação tem se modificado ao longo dos anos. Ainda assim, pode-se perceber o aumento da evasão e do desinteresse por parte dos alunos. Devido às tarefas de Engenharia de Software serem, muitas vezes, consideradas repetitivas, ensiná-las pode ser um desafio, uma vez que os alunos podem se sentir desmotivados. Para transformar um assunto difícil em algo interessante, a gamificação tem sido então utilizada [Alhammad and Moreno 2018]. A gamificação é o "uso de elementos de design de jogos em contextos não relacionados a jogos" [Deterding et al. 2011]. Um dos objetivos da sua implementação é associar a uma atividade, os mesmos elementos que tornam os jogos interessantes e divertidos [Fardo 2013]. Aplicar a gamificação no ensino e aprendizagem de ES pode ser uma solução interessante ([Pedreira et al. 2015, Alhammad and Moreno 2018, Mauricio et al. 2018, Klock et al. 2018, Jesus et al. 2018]).

Diante disso, o objetivo desse artigo é caracterizar propostas existentes, identificadas a partir de um mapeamento sistemático, sobre o uso da gamificação no ensino e aprendizagem de ES, bem como analisar suas aplicações e resultados. Das 617 publicações 
encontradas, 17 foram selecionadas para responder às questões de pesquisa propostas. Vale destacar que este mapeamento tem como foco práticas de gamificação no contexto acadêmico. Publicações em contextos profissionais não foram incluídas. Para tanto, este artigo está organizado como a seguir. A Seção 2 apresenta a metodologia usada no mapeamento. A Seção 3 analisa as questões de pesquisa e apresenta os resultados obtidos. A Seção 4 apresenta trabalhos relacionados a esta proposta. A Seção 5 se refere às ameaças à validade desta proposta e a Seção 6 discute as considerações finais e os trabalhos futuros.

\section{Mapeamento Sistemático}

O mapeamento sistemático é um meio de descobrir, avaliar e interpretar as pesquisas disponíveis e relevantes sobre uma questão de pesquisa para identificar lacunas nas pesquisas atuais e sugerir uma investigação mais aprofundada [Kitchenham 2004]. O processo do mapeamento descrito neste artigo foi conduzido por alunos de graduação com o auxílio do Parsif.al ${ }^{1}$, como a seguir. A etapa de planejamento consiste em especificar e avaliar o protocolo a ser seguido. O protocolo apresenta o objetivo do mapeamento, as questões de pesquisa, a definição das fontes de pesquisa, a string de busca e os critérios de exclusão das publicações obtidas. Terminada esta etapa, a condução do mapeamento é iniciada. Durante essa fase, as publicações são identificadas a partir da estratégia de busca definida e os critérios de exclusão são aplicados. A seguir, os dados das publicações selecionadas são extraídos e sintetizados para responder as questões de pesquisa e facilitar as análises e síntese dos resultados. Para finalizar, os resultados apresentados.

Com base no objetivo definido, foram elaboradas as questões de pesquisa (QP):

- QP1: Em que contextos da disciplina de Engenharia de Software a gamificação tem sido aplicada? Com essa questão, procura-se entender em quais tipos de cursos e processos de ES foram implementados métodos de gamificação;

- QP2: Como a gamificação foi implementada para apoiar o ensino e aprendizagem de Engenharia de Software? Esta questão busca compreender como a gamificação foi trabalhada no currículo da disciplina de ES. Esses dados são usados para coletar informações sobre os elementos de gamificação usados;

- QP3: Que tipos de ferramentas foram usados para implementar a solução gamificada? Avalia-se os instrumentos usados para aplicar a gamificação, bem como, se um novo software foi desenvolvido ou se uma plataforma de gamificação já existente ou um software sem elementos de gamificação foram usados;

- QP4: Qual é o impacto evidencial da gamificação no ensino e aprendizagem de Engenharia de Software? Com essa questão de pesquisa, busca-se os aspectos positivos e negativos da aplicação dos elementos de gamificação no ensino e aprendizagem de ES, bem como os motivos por trás dos resultados alcançados;

- QP5: Que tipo de avaliação foi efetuada para verificar o impacto da gamificação no ensino e aprendizagem de ES? Busca-se apontar os estudos experimentais, avaliações qualitativas e quantitativas para medir tal impacto;

- QP6: Quais são os autores mais ativos na área? Esta questão de pesquisa pretende apontar os principais pesquisadores da área;

- QP7: Quais são os países que mais realizaram publicações sobre o tema? Esta questão de pesquisa pretende apontar como estão as pesquisas no mundo e especialmente no Brasil para possíveis colaborações;

${ }^{1}$ https://parsif.al/ 
IX Congresso Brasileiro de Informática na Educação (CBIE 2020)

Anais do XXXI Simpósio Brasileiro de Informática na Educação (SBIE 2020)

- QP8: Quais os principais meios de divulgação das publicações? Com esta questão, pretende-se identificar veículos para futuras análises relacionadas à utilização da gamificação no ensino e aprendizagem de Engenharia de Software.

O próximo passo foi definir as fontes de pesquisa (bibliotecas digitais) para seleção das publicações. Foram escolhidas Ei Compendex, IEEExplore e Scopus a partir dos critérios: capacidade de usar operadores lógicos (ORs e ANDs) no processo de busca; suporte a pesquisas completas ou por área; disponibilidade na instituição dos autores; abrangência da área de Ciência da Computação [Costa and Murta 2013].

Para definir a string de busca (Tabela 1), foi realizado um processo de teste e refinamento adaptado do método PICOC [Kitchenham and Charters 2007]: População (software engineering), Intervenção (gamification), Comparação, Output/Resultado e Contexto (educat*) . Como o objetivo deste mapeamento é caracterizar as abordagens na área de pesquisa, a comparação não se aplica e o resultado não foi aplicado, seguindo o que foi feito nos mapeamentos relacionados (Seção 4). Vale mencionar que a revisão da string foi realizada por especialistas do domínio e todos os termos sinônimos da instanciação do PICOC foram extraídos de [Sommerville 2011]. Além disso, a validação da string foi feita através de três artigos de controle [Pedreira et al. 2015, Berkling and Thomas 2013, Dubois and Tamburrelli 2013] e de sua verificação no retorno nas buscas. Uma revisão da literatura convencional anterior obteve tais artigos de controle. Eles foram úteis para fornecer uma compreensão inicial da área bem como para definir a string de busca.

Tabela 1. String de busca

(gamification OR gamify OR gamifying OR gamified OR funware) AND ("software engineering"OR "software process"OR "requirements engineering"OR "system modeling"OR "software testing"OR "project planning"OR "configuration management"OR "software evolution"OR "architectural design"OR "design and implementation"OR "quality management"OR "project management"OR "agile software development"OR "software reuse"OR "component-based software engineering"OR "distributed software engineering"OR "service-oriented software engineering"OR "systems engineering"OR "systems of systems"OR "real-time software engineering"OR "dependable systems"OR "reliability engineering"OR "safety engineering"OR "security engineering"OR "resilience engineering") AND (educat* OR course OR teach* OR learn* OR train* OR curricul* OR syllab*)

A Tabela 2 descreve os critérios de exclusão (CE) [Petersen et al. 2008] que verificam se uma publicação tem potencial ou não para compor este mapeamento.

Tabela 2. Critérios de Exclusão

\footnotetext{
CE1 Estudos que não tratam da gamificação no contexto da educação na disciplina de ES

CE2 Estudos não escritos em inglês ou português

CE3 Estudos não totalmente disponíveis

CE4 Teses, dissertações, capítulos de livros, palestras, tutoriais, surveys, mapeamentos e sumários

CE5 Estudos com versões mais recentes
}

Já a Tabela 3 apresenta os resultados obtidos com a string de busca adaptada para cada biblioteca e a pesquisa sobre o título, o resumo e as palavras-chave. Na primeira coluna, tem-se as bibliotecas usadas; na segunda, o total de publicações e na terceira, como muitas publicações estão em mais de uma biblioteca, as duplicatas foram eliminadas. 
IX Congresso Brasileiro de Informática na Educação (CBIE 2020)

Anais do XXXI Simpósio Brasileiro de Informática na Educação (SBIE 2020)

Tabela 3. Total de publicações por biblioteca digital

\begin{tabular}{ccc}
\hline Bibliotecas & Publicações & Sem Duplicatas \\
\hline Ei Compendex & 267 & 149 \\
IEEExplore & 100 & 56 \\
Scopus & 250 & 121 \\
Total & $\mathbf{6 1 7}$ & $\mathbf{3 2 6}$ \\
\hline
\end{tabular}

Em seguida, foi executada uma primeira seleção (Etapa 1), que aplicou, manualmente, os critérios de exclusão no título, resumo e palavras-chave nas 326 publicações. Nesta etapa, 282 publicações foram eliminadas (Tabela 4). Em uma segunda avaliação das 44 publicações restantes (Etapa 2), aplicou-se os critérios também na introdução e conclusão. Por fim, uma última avaliação (Etapa 3), aplicou os critérios em todo o conteúdo das 30 publicações. Este processo foi então finalizado com 17 publicações resultantes.

Tabela 4. Total de publicações excluídas por critério

\begin{tabular}{cccc}
\hline Critério & Etapa 1 & Etapa 2 & Etapa 3 \\
\hline CE1 & 214 & 12 & 11 \\
CE2 & 10 & 1 & 0 \\
CE3 & 0 & 0 & 0 \\
CE4 & 58 & 1 & 1 \\
CE5 & 0 & 0 & 1 \\
Total & $\mathbf{2 8 2}$ & $\mathbf{1 4}$ & $\mathbf{1 3}$ \\
\hline
\end{tabular}

\section{Resultados}

As 17 publicações resultantes (Tabela 5) foram selecionadas para responder as questões de pesquisa a seguir. A questão (QP1) tem como objetivo descobrir que tipo de curso e quais processos de ES tem sido alvo da gamificação e os motivos para tal. Em geral, a gamificação vem sido aplicada em turmas presenciais de ES ([P2], [P4], [P5], [P6], [P8], [P9], [P10], [P11], [P12], [P14] e [P17]) ou em suas vertentes, como Projeto de Software ([P1]), Design e Desenvolvimento de Software ([P13]), Gerenciamento de Riscos ([P7]) e Engenharia de Sistemas ([P15]). Em [P3], a aplicação é em uma turma online de Engenharia de Requisitos e em [P16] no trabalho de conclusão de curso. Vale destacar que [P2] e [P5] aplicaram gamificação em cursos onde os alunos conciliavam a faculdade com trabalhos remunerados e [P4] propõe o uso da gamificação nesse mesmo ambiente.

A questão QP2 identifica os métodos utilizados para integrar a gamificação na disciplina de ES e suas vertentes. Uma das publicações menciona a utilização da gamificação de forma implícita ([P5]). Em [P4], o autor afirma que seu uso explícito pode gerar a ideia de "não-seriedade" aos alunos. Além disso, um autor não implementa a solução proposta ([P7]). Já [P12] mostra a implementação de 8 tipos de medalhas. Em [P15], foi desenvolvido um design de instrumento pedagógico (PID) sob PSP baseado em gamificação para criar cenários lúdicos em sala de aula. Em [P17], além do role-play também foram usados jogos sérios. A implementação de elementos de jogos no ensino funciona como motivação para os alunos concluírem uma tarefa de estudo. Quando a motivação vem de um elemento externo, ou seja, a partir, por exemplo, da conclusão de um objetivo apresentado 
IX Congresso Brasileiro de Informática na Educação (CBIE 2020)

Anais do XXXI Simpósio Brasileiro de Informática na Educação (SBIE 2020)

Tabela 5. Referências para as Publicações

\begin{tabular}{ll}
\hline P\# - Referência & P\# - Referência \\
\hline P1 - [Singer and Schneider 2012] & P2 - [Berkling and Thomas 2013] \\
P3 - [Dubois and Tamburrelli 2013] & P4 - [Thomas and Berkling 2013] \\
P5 - [Berkling 2015] & P6 - [Laskowski 2015] \\
P7 - [Uyaguari et al. 2015] & P8 - [Fu and Clarke 2016] \\
P9 - [Mora et al. 2016] & P10 - [Su 2016] \\
P11 - [Unkelos-Shpigel 2016] & P12 - [Souza et al. 2017] \\
P13 - [Matsubara and Da Silva 2017] & P14 - [Vasconcelos et al. 2018] \\
P15 - [Gasca-Hurtado et al. 2019] & P16 - [Al-Azawi et al. 2019] \\
P17 - [Ivanova et al. 2019] & \\
\hline
\end{tabular}

pelo curso, é nomeada de extrínseca [Hanus and Fox 2015]. Dentre os elementos de maior destaque, point, leaderboard, competition e badge tem característica extrínseca quanto à motivação que provocam. Por outro lado, a motivação intrínseca [Hanus and Fox 2015] é caracterizada pelo fato de a motivação partir do aluno, sem a necessidade de um fator externo. Já a questão QP3 identifica as ferramentas utilizadas na gamificação e as suas contribuições. Uma publicação não explicita a ferramenta utilizada ([P5]) e outra apresenta uma sugestão de gamificação (sem implementá-la) ([P7]). Algumas fazem uso do Moodle ([P9]) e CourseSites ${ }^{2}$ ([P4]). Em certos casos, os próprios autores implementaram as plataformas: WReSTT-CyLE ([P8]), GSEELS (Sistema de Educação e Aprendizado de Engenharia de Software Gamificado) ([P10]). Outras aplicações e frameworks não foram nomeados ([P5] e [P16]). Um dos ambientes foi implementado com o auxílio do framework Vaadin ([P2]). Em ([P15]), foi implementado um Design de Instrumento Pedagógico sob PSP com a temática de design de carros sendo comparada com o design de software. Outras ferramentas são apresentadas na Tabela 6.

Tabela 6. Ferramentas utilizadas

\begin{tabular}{lll}
\hline Ferramenta & Endereço Eletrônico & Referência \\
\hline Subversion & https://subversion.apache.org/ & {$[\mathrm{P} 1]$} \\
Trac & https://trac.edgewall.org/ & {$[\mathrm{P} 1]$} \\
Trello & https://trello.com/ & {$[\mathrm{P} 3],[\mathrm{P} 14]$} \\
Google Forms & https://www.google.com/forms/about/ & {$[\mathrm{P} 11],[\mathrm{P} 12]$} \\
Google Spreadsheet & https://www.google.com/sheets/about/ & {$[\mathrm{P} 6]$} \\
Kahoot! & https://kahoot.com/ & {$[\mathrm{P} 11],[\mathrm{P} 17]$} \\
LevelLearn & http://www.levellearn.com.br/ & {$[\mathrm{P} 14]$} \\
Sonar & https://www.sonarqube.org & {$[\mathrm{P} 13]$} \\
\hline
\end{tabular}

A questão QP4 analisa o impacto da gamificação na disciplina e os resultados por meio de feedback, notas, comparações com versões não-gamificadas, entre outros. Em [P4] e [P7], as propostas não são implementadas. Várias propostas revelam a gamificação como motivadora. Em [P1], [P9] e [P14] a motivação se deu por conta da natureza competitiva e pelo objetivo de não ficar em último, gerando certa pressão aos alunos e, possivelmente, sendo um elemento negativo. Quanto ao feedback dos alunos, as propostas

\footnotetext{
${ }^{2}$ https: / / coursesites.com/
} 
foram bem recebidas, mas com algumas críticas, relacionadas aos elementos implementados ([P9]), ao caráter visual da plataforma ([P2], [P5] e [P9]) ou ao método de pontuação ([P1]). Em [P3], o autor conclui que o processo é benéfico sem medi-lo. Ao ser aplicada em alunos que não possuem o hábito de jogar, a gamificação explícita não se mostrou um elemento motivador ([P2]). Quanto às notas, alguns alunos que participaram do processo de gamificação obtiveram notas inferiores aos que não participaram ([P6]). Em outro cenário, os alunos submetidos à gamificação conseguiram notas melhores e menor índice de reprovação ([P13]). Em [P8], alunos obtiveram nota melhor, mas o foco ficou em ganhar pontos ao invés de aprender. Em [P15], os participantes disseram que aplicariam PSP na sua área de trabalho por entender sua importância. Os membros da equipe gamificada de [P16] obtiveram notas melhores no projeto de último ano em relação aos outros anos não gamificados. Os alunos de [P17] revelaram que seu interesse e motivação para aprender melhoraram após a participação nas atividades gamificadas. Já [P10], [P11] e [P12] confirmaram o experimento como positivo, mas sem descrever detalhes. Já a questão QP5 analisa como foi realizada a avaliação do impacto da gamificação no ensino e aprendizagem de ES. O processo mais comum foi a aplicação de questionários ([P1], [P2], [P5], [P9], [P10], [P11], [P12], [P14], [P15] e [P17]). As notas dos alunos também foram comparadas em [P6] e [P8]. Em [P13], foi feita uma pesquisa quantitativa. Dois outros estudos [P6] e [P8] avaliaram o engajamento das turmas. Uma publicação utilizou métricas do Sonar ([P3]). Como já mencionado, duas publicações não implementaram a gamificação ([P4] e [P7]). Já a publicação [P16] não cita o processo utilizado.

A questão QP6 busca os autores que mais publicaram. Poucos autores tem mais de uma publicação: Kay Berkling ([P2], [P4] e [P5]) e Christoph Thomas ([P2] e [P4]. Já a questão QP7 tem como objetivo identificar os países com mais publicações. As pesquisas estão distribuídas entre Alemanha ([P1], [P2], [P4] e [P5]), Brasil ([P12], [P13] e [P14]) e os demais países com uma publicação cada: EUA ([P8]), Polônia ([P6], Israel ([P11]), Rússia ([P3]), Espanha ([P9]), Equador ([P7]), Taiwan ([P10]), Colômbia ([P15]), Omã [P16] e Croácia ([P17]). A presença do Brasil nas questões QP6 e QP7 traz resultados desejados do mapeamento: descobrir pesquisadores para futuras colaborações. Por último, a questão QP8 identifica os meios onde as publicações foram divulgadas. Apenas um trabalho foi publicado em um journal e os outros em conferências (Tabela 7). As conferências AISC e ICL divulgaram duas publicações cada e as demais, apenas uma.

Tabela 7. Meios de divulgação das publicações

\begin{tabular}{llcc}
\hline Tipo & Lugares das publicações & Qtde & $\mathbf{\%}$ \\
\hline Conferência & AISC [P7] [P14], ICL [P2] [P4], ASEE [P8], CCIS [P5], CSEE\&T [P12], & 16 & $\mathbf{9 4 \%}$ \\
& EDUCON [P6], ESEC/FSE [P3], GAS [P1], ICSE-SEET [P13], LNBIP & \\
& [P11], TEEM [P9], WorldCIST [P15], LTEC [P16], MIPRO [P17] & \\
Journal & Multimedia Tools and Applications [P10] & 1 & $\mathbf{6 \%}$ \\
\hline
\end{tabular}

\section{Trabalhos Relacionados}

Essa seção apresenta alguns mapeamentos relacionados à proposta deste artigo. O mapeamento apresentado em [Pedreira et al. 2015] tem como objetivo caracterizar o estado da arte do uso de gamificação em ES. O estudo foi efetuado nas bibliotecas Scopus, ScienceDirect, Wiley InterScience, IEEExplore, ACM e Springer, sendo limitado a publicações 
até junho de 2014. Depois do processo de exclusão, 29 publicações foram selecionadas. Entre os elementos de jogos, ponto foi o mais adotado dentre as publicações analisadas, seguido pelo uso de badges e ranques [Pedreira et al. 2015]. Em [Klock et al. 2018], um mapeamento com ênfase na avaliação da gamificação em ambientes educacionais foi descrito. O estudo efetuado em 2015 na ACM, IEEExplore, ScienceDirect, Scopus, Springer e Web of Science considerou publicações de 2010 em diante. Medalhas, além dos leaderboards e pontos foram os elementos de jogos mais utilizados dentre as 20 publicações resultantes. Já o mapeamento descrito em [Jesus et al. 2018] foi executado em 2018 na Scopus e trata da gamificação em teste de software. Foram selecionadas 15 publicações de gamificação no ensino de teste e na sua execução. Entre seus resultados, pontos, leaderboards e níveis foram os elementos mais utilizados e os objetivos mais comuns foram aumentar o engajamento, a motivação e a satisfação dos alunos.

Em [Mauricio et al. 2018], os autores apresentam um mapeamento relacionado ao uso de jogos e elementos de jogos na educação de Engenharia de Software. O estudo foi realizado em 2016 utilizando ACM, IEEExplore, Ei Compendex e Scopus. Dentre os 156 estudos primários retornados, 10 publicações tratam do uso de gamificação no ensino de ES e uma publicação de 1974 com o assunto de GDBL $^{3}$ (Game Development Based Learning) foi recuperada. Em paralelo, a primeira publicação de gamificação é de 2011. Já o mapeamento apresentado em [Alhammad and Moreno 2018] tem como objetivos identificar em quais contextos a gamificação estava sendo aplicada no ensino de Engenharia de Software, como ela foi aplicada e qual o seu impacto evidencial. Dentre os 21 estudos resultantes, 15 aplicaram gamificação em cursos tradicionais, sem parte online, 1 em um curso todo online e 5 em cursos mistos, com a parte gamificada online. Os autores utilizam as definições de dinâmicas, mecânicas e componentes apresentadas em [Werbach and Hunter 2015]. Os elementos desafios, feedback e recompensas e os componentes quadro de líderes, pontos e níveis foram os mais utilizados.

Concluindo, o mapeamento descrito neste artigo, assim como os demais desta seção, apresentam em sua maioria resultados positivos da aplicação da gamificação. Além disso, ainda não existe uma abordagem sistemática para aplicação da gamificação na ES e em função disso a necessidade de mais pesquisas com este propósito. Por outro lado, este mapeamento analisa propostas de gamificação exclusivamente no contexto acadêmico. Já [Pedreira et al. 2015] analisou experiências em geral (contexto profissional e acadêmico) mas excluiu os estudos com objetivo principal de gamificação aplicada à educação. Outro ponto é que o mapeamento descrito em [Jesus et al. 2018] está relacionado à gamificação em teste de software, enquanto este mapeamento (e os demais descritos nesta seção) incluem todas as áreas da ES. Para finalizar, este mapeamento foi concluído no primeiro trimestre deste ano e apresenta uma atualização temporal quando comparado aos demais e inclui em seus resultados pesquisas mais recentes [Vasconcelos et al. 2018, Gasca-Hurtado et al. 2019, Al-Azawi et al. 2019, Ivanova et al. 2019].

\section{Ameaças à Validade}

Durante o mapeamento, buscou-se evitar ameaças que pudessem limitar a validade dos resultados [Wohlin et al. 2012]. No entanto, não é possível garantir que tais ameaças, entre outras, não tenham afetado os resultados. As etapas de seleção e extração de dados

\footnotetext{
${ }^{3}$ www.igi-global.com/dictionary/game-development-based-learning/60064
} 
foram realizadas de forma independente por 4 autores, divididos em 2 grupos, para diminuir a subjetividade. Os resultados individuais eram conferidos pelo par e, caso ocorresse divergência, os autores discutiam os motivos de suas decisões para chegar a um consenso, relendo as publicações se necessário. Problemas na string de busca podem ter levado à ausência de algumas publicações. Para minimizar, a string foi testada e alterada em várias etapas. Além disso, considerar apenas 3 bibliotecas, apesar de alguns critérios estabelecidos (Seção 2), pode significar não indexar todo o conteúdo disponível na Web. Por este motivo é possível que algumas publicações relevantes não tenham sido contempladas. Finalizando, este mapeamento foi concluído em março de 2020. Assim sendo, versões recentes de trabalhos ou avanços de pesquisas podem não ter sido contemplados.

\section{Considerações Finais}

O mapeamento descrito neste artigo tem como objetivo detectar as principais características presentes em abordagens de gamificação no apoio ao ensino e aprendizagem de ES, considerando o estado da arte. A partir do mapeamento concluiu-se que a aplicação da gamificação apresenta resultados positivos como o aumento da motivação dos alunos ([P1], [P3], [P5], [P9], [P14] e [P17]) e de suas notas ([P8], [P13] e [P16]) bem como negativos ([P2]), devido a natureza competitiva do processo, o que desmotivou os alunos.

Como trabalhos futuros, pretende-se aplicar uma busca manual e um snowballing [Wohlin et al. 2012], para atualizar e ampliar o conhecimento sobre a área. Além disso, a partir dos resultados deste mapeamento, uma proposta de gamificação da disciplina de ES do Departamento de Ciência da Computação da Universidade Federal de Juiz de Fora está sendo desenvolvida e uma avaliação inicial foi efetuada [Carvalho et al. 2020]. Nesta primeira versão de gamificação da disciplina, os alunos foram divididos em grupos de 5 e tinham como objetivo inicial implementar um website para um supermercado fictício. Outras atividades da disciplina como escrita de artigo e exercícios em sala de aula foram gamificados. Completar as tarefas dentro do prazo, dividir as tarefas coletivas entres os integrantes dos grupos, apresentar atividades e a presença em sala eram recompensadas com pontos. Na página da disciplina, após o término do projeto, foi disponibilizada, anonimamente, a pontuação dos três melhores alunos. Após a conclusão da disciplina, um formulário de feedback foi aplicado. A maioria dos alunos se sentiu bem em um cenário competitivo e aprovaram o uso da gamificação na disciplina. Por outro lado, alguns disseram que eram indiferentes ou discordavam da afirmação de se sentirem motivados para conseguir as recompensas. Uma segunda versão da proposta se encontra em fase de desenvolvimento. Nesta versão, algumas sugestões apresentadas no formulário de feedback e resultados deste mapeamento serão implementadas, tais como o sistema de ranking. Além disso, novas atividades e recompensas serão elaboradas para manter o aluno motivado durante todo o semestre letivo. Ao final, pretende-se realizar um estudo experimental para que seja verificado o impacto no ensino e aprendizagem da disciplina.

\section{Referências}

Al-Azawi, R., Joe, S. A., Al-Obaidy, M., and Westlake, J. (2019). The use of gamification technique in agile development methodology. In International Workshop on Learning Technology for Education in Cloud, pages 3-13. Springer.

Alhammad, M. M. and Moreno, A. M. (2018). Gamification in software engineering education: A systematic mapping. In Systems and Software, pages 131-150. Elsevier. 
IX Congresso Brasileiro de Informática na Educação (CBIE 2020)

Anais do XXXI Simpósio Brasileiro de Informática na Educação (SBIE 2020)

Berkling, K. (2015). Gamification behind the scenes. In International Conference on Computer Supported Education, pages 274-292. Springer.

Berkling, K. and Thomas, C. (2013). Gamification of a software engineering course and a detailed analysis of the factors that lead to it's failure. In 2013 International Conference on Interactive Collaborative Learning (ICL), pages 525-530. IEEE.

Carvalho, D., Altomar, M., Furtado, F., and Oliveira, A. (2020). Proposta de gamificação aplicada ao ensino e aprendizagem de engenharia de software. In submitted.

Costa, C. and Murta, L. (2013). Version control in distributed software development: A systematic mapping study. In Global Software Engineering (ICGSE), 2013 IEEE 8th International Conference on, pages 90-99. IEEE.

Deterding, S., Dixon, D., Khaled, R., and Nacke, L. (2011). From game design elements to gamefulness: defining gamification. In Proceedings of the 15th Intern. Academic MindTrek Conference: Envisioning future media environments, pages 9-15. ACM.

Dubois, D. J. and Tamburrelli, G. (2013). Understanding gamification mechanisms for software development. In Proceedings of the 2013 9th Joint Meeting on Foundations of Software Engineering, pages 659-662. ACM.

Fardo, M. L. (2013). A gamificação aplicada em ambientes de aprendizagem. In RENOTE-Revista Novas Tecnologias na Educação, 11. RENOTE v. 11 n. 1.

Fu, Y. and Clarke, P. (2016). Gamification-based cyber-enabled learning environment of software testing. In submitted to the 123rd - ASEE - Software Eng. Constituent. IEEE.

Gasca-Hurtado, G. P., Gómez-Álvarez, M. C., and Manrique-Losada, B. (2019). Using gamification in software engineering teaching: Study case for software design. In World Conference on Information Systems and Technologies, pages 244-255. Springer.

Hanus, M. D. and Fox, J. (2015). Assessing the effects of gamification in the classroom: A longitudinal study on intrinsic motivation, social comparison, satisfaction, effort, and academic performance. Computers \& education, 80:152-161.

Ivanova, G., Kozov, V., and Zlatarov, P. (2019). Gamification in software engineering education. In 201942 nd International Convention on Information and Communication Technology, Electronics and Microelectronics (MIPRO), pages 1445-1450. IEEE.

Jesus, G. M., Ferrari, F. C., de Paula Porto, D., and Fabbri, S. C. P. F. (2018). Gamification in software testing: A characterization study. In Proceedings of the III Brazilian Symposium on Systematic and Automated Software Testing, pages 39-48. ACM.

Kitchenham, B. (2004). Procedures for performing systematic reviews. In Keele, UK, Keele University, volume 33, pages 1-26. Keele, UK, Keele University.

Kitchenham, B. and Charters, S. (2007). Guidelines for performing systematic literature reviews in software engineering.

Klock, A. C. T., Ogawa, A. N., Gasparini, I., and Pimenta, M. S. (2018). Does gamification matter?: a systematic mapping about the evaluation of gamification in educational environments. In Proc. ACM Symposium on Applied Computing, pages 2006-2012.

Laskowski, M. (2015). Implementing gamification techniques into university study path-a case study. In Global Engineering Education Conference, pages 582-586. IEEE. 
IX Congresso Brasileiro de Informática na Educação (CBIE 2020)

Anais do XXXI Simpósio Brasileiro de Informática na Educação (SBIE 2020)

Matsubara, P. G. F. and Da Silva, C. L. C. (2017). Game elements in a software engineering study group: a case study. In Proceedings of the 39th Intern. Conf. on Software Engineering: Software Engineering and Education Track, pages 160-169. IEEE Press.

Mauricio, R. d. A., Veado, L., Moreira, R. T., Figueiredo, E., and Costa, H. (2018). A systematic mapping study on game-related methods for software engineering education. Information and software technology, 95:201-218.

Mora, A., Planas, E., and Arnedo-Moreno, J. (2016). Designing game-like activities to engage adult learners in higher education. In Proceedings of the Fourth Intern. Conf. on Technological Ecosystems for Enhancing Multiculturality, pages 755-762. ACM.

Pedreira, O., García, F., Brisaboa, N., and Piattini, M. (2015). Gamification in software engineering-a systematic mapping. In Information and Software Technology, volume 57, pages 157-168. Elsevier.

Petersen, K., Feldt, R., Mujtaba, S., and Mattsson, M. (2008). Systematic mapping studies in software engineering. In 12th International Conference on Evaluation and Assessment in Software Engineering (EASE) 12, volume 8, pages 68-77. EASE.

Singer, L. and Schneider, K. (2012). It was a bit of a race: Gamification of version control. In 2012 Second Intern. Workshop on Games and Software Engineering: Realizing User Engagement with Game Engineering Techniques (GAS), pages 5-8. IEEE.

Sommerville, I. (2011). Software engineering 9th edition. In ISBN-10137035152. ISBN0.

Souza, M., Veado, L., Constantino, K., and Figueiredo, E. (2017). Gamification in software engineering education: An empirical study. In Software Engineering Education and Training, pages 276-284. IEEE 30th Conference.

$\mathrm{Su}$, C. H. (2016). The effects of students' motivation, cognitive load and learning anxiety in gamification software engineering education: a structural equation modeling study. In Multimedia Tools and Applications, volume 75, pages 10013-10036. Springer.

Thomas, C. and Berkling, K. (2013). Redesign of a gamified software engineering course. In 2013 Intern. Conf. on Interactive Collaborative Learning, pages 778-786. IEEE.

Unkelos-Shpigel, N. (2016). Peel the onion: Use of collaborative and gamified tools to enhance software engineering education. In International Conference on Advanced Information Systems Engineering, pages 122-128. Springer.

Uyaguari, F. U., Intriago, M., and Jácome, E. S. (2015). Gamification proposal for a software engineering risk management course. In New Contributions in Information Systems and Technologies, pages 795-802. Springer.

Vasconcelos, L. E., Oliveira, L., Guimarães, G., and Ayres, F. (2018). Gamification applied in the teaching of agile scrum methodology. In Information Technology - New Generations, pages 207-212. Springer.

Werbach, K. and Hunter, D. (2015). The gamification toolkit: dynamics, mechanics, and components for the win. Wharton School Press.

Wohlin, C., Runeson, P., Höst, M., Ohlsson, M. C., Regnell, B., and Wesslén, A. (2012). Experimentation in software engineering. Springer Science \& Business Media. 